\title{
Gauss-Simpson Quadrature Algorithm for Calcaulting Additional Stress in Foundation Soils
}

Shuai WANG ( $\square$ wangshuai@Intu.edu.cn )

Liaoning Technical University

Chao WANG

Liaoning Technical University

Chenliang XING

Liaoning Technical University

\section{Research Article}

Keywords: Additional stress in soils, Additional foundation pressure, Gaussian quadrature formula, Simpson quadrature formula, Elastic theory, Flexible foundation, Numerical integration calculation

Posted Date: July 8th, 2021

DOI: https://doi.org/10.21203/rs.3.rs-657109/v1

License: @ (i) This work is licensed under a Creative Commons Attribution 4.0 International License. Read Full License 
1 Gauss-Simpson Quadrature Algorithm for Calcaulting Additional Stress in Foundation Soils

2 Shuai WANG ${ }^{\mathrm{a}, *},($ E-mail address: wangshuai@lntu.edu.cn)

3 a School of Civil Engineering, Liaoning Technical University, Fuxin Liaoning, 123000, China

4 Chao $\mathrm{WANG}^{\mathrm{b}}$ (E-mail address:1159704386@qq.com)

$5 \quad{ }^{\mathrm{b}}$ School of Civil Engineering, Liaoning Technical University, Fuxin Liaoning, 123000, China

6 Chenliang XING ${ }^{\mathrm{c}}$ (E-mail address:1434395793@qq.com)

$7 \quad$ c School of Civil Engineering, Liaoning Technical University, Fuxin Liaoning, 123000, China

$8 \quad *$ Corresponding author:

9 Shuai WANG ${ }^{\mathrm{a}}$ E-mail address: wangshuai@Intu.edu.cn (S. WANG).

10 Abstract:

11 The additional pressure at the bottom of a building's foundation produces an additional stress in

12 the foundation soils under the building's foundation. In order to overcome the limitations of

13 traditional elastic theory methods and the finite element method when calculating the additional

14 stress in foundation soils, we use the Gauss-Simpson formula to derive the Gauss-Simpson

15 Quadrature Algorithm based on the elasticity theory. The Gauss-Simpson Quadrature Algorithm

16 is a method designed to calculate the additional stress in foundation soils under an irregularly

17 shaped foundation and an irregular load distribution. This new method is based on the fact that the

18 Gaussian quadrature formula and the Simpson formula are independent of the specific type of

19 integrand. The finite element method with $\mathrm{n}$ interpolation points can only achieve an algebraic

20 accuracy of $\mathrm{n}$. The interpolation points of the Gaussian quadrature formula are $\mathrm{n}$ zeros of 
21 orthogonal polynomials, which can achieve an algebraic accuracy of $2 n+1$. Moreover, the weights

22 of the nodes in the quadrature formula are all positive, and thus, it has a high numerical stability.

23 In the proposed method, the Simpson formula is necessary. The Simpson formula is used to

24 transform the implicit additional stress formula with the integral sign into an explicit cumulative

25 integral, which can be considered similar to the rectangular domain case to obtain an explicit

26 analytical algebraic formula for solving the additional stress approximation. In engineering

27 applications, we only need to provide the field engineers with the locations of the interpolation

28 points of the Gauss-Legendre formula, the interpolated weight coefficients, and the specific type

29 of Simpson's formula, and then, the results of the additional stress can be calculated manually,

30 which is nearly impossible using the traditional methods and finite element methods. From the

31 point of view of academic rigor and theoretical completeness, it is possible to use the compound

32 Gauss-Simpson Quadrature Algorithm in conjunction with the looping function in computer

33 programs. Under standard conditions, the proposed Gauss-Simpson Quadrature Algorithm is in

34 good agreement with the results of the traditional elasticity theory.

35 Keywords:

36

37

38
Additional stress in soils; Additional foundation pressure; Gaussian quadrature formula; Simpson quadrature formula; Elastic theory; Flexible foundation; Numerical integration calculation

\section{Introduction}

Additional stress in foundation soils leads to deformation of the foundation soil and causes building settlement [1-2]. Currently, the calculation of additional stress on a foundation is based on the elastic theory, which assumes that the foundation soil is a homogeneous, continuous, 
isotropic, semi-infinite spatial elastomer [3]. According to the Flamant solution and the Boussinesq solution in classical elastic mechanics [3], the additional stress in the foundation soil induced by a concentrated load, a uniform load, a triangular load, and a trapezoidal load can be obtained for strip, rectangular, and circular foundations [4]. Using the vertical stress induction diagram developed by Newmark in 1942 [4], it is possible to calculate the additional stress in an irregularly shaped foundation under a uniformly distributed load [5-6].

In practical engineering, there are numerous cases with irregular load distributions, and the uniform load assumption is a simple and rough averaging method, which is an expedient way to deal with the problem. In traditional methods, the most widely used stress distributions are rectangular and circular, which have difficulty meeting the requirements of modern architecture and artistic aesthetics.

The type and shape of the load distribution are not regular in practical engineering [7-8]. The traditional elastic theory used to calculate the additional stress of the foundation is limited by the integrability of the integrand, and it can only be used to calculate the additional stress on a foundation with a regular shape and a uniformly distributed load. Therefore, it is of major theoretical and engineering significance to develop a method of calculating the additional stress in an irregularly shaped foundation under an irregular load distribution.

From the perspective of the additional pressure at the bottom of the foundation, it is an irregular load distribution, and its distribution shape is irregular [9-12]. Therefore, the application of the traditional calculation method is largely limited. The uniform load assumption in practical engineering is a simple averaging method, and it is a limitation of traditional calculation methods, including the Newmark chart method.

In addition, the most widely used types of stress distribution are rectangular and circular, 
which cannot meet the requirements of modern architecture with artistic aesthetics. Currently, the most widely used mainstream method used to solve the problem of an irregular load distribution and an irregular distribution shape is FE simulations. Therefore, the method proposed in this study must have significant advantages over the finite element method in order to have value for practical applications.

The method proposed in this study has something in common with the finite element method. Specifically, both are based on nodal interpolation functions that approximate the stress distribution functions, including the stress magnitude distribution and the shape of the loaded region. The essence of the finite element method is to use the displacement interpolation function to approximate the original function, and it is a commonly used interpolation method. It can usually only achieve algebraic accuracy equal to the number of interpolations. The ususal method of improving the calculation accuracy is to increase the number of interpolations, which results in a higher risk of Runge phenomenon and thus an unstable calculation. Therefore, for large-scale finite element simulations, the usual solution is to use as many low order $(<4)$ elements as possible to avoid mesh distortion and numerical instability. This method greatly increases the number of elements and the computational amount, resulting in a high computational cost and low computational efficiency.

In view of the limitations of the traditional elasticity theory method and FE simulation methods, the Gauss-Simpson Quadrature Algorithm was applied to calculate the additional stress in the soil under a foundation based on the fact that the Gauss-Simpson formula is independent of the specific type of the integrand. In addition, the equation for caculating the additional stress was derived based on elasticity theory. The finite element method with $n$ interpolation points can only achieve an algebraic accuracy of $n$. The interpolation points of the Gaussian quadrature formula 


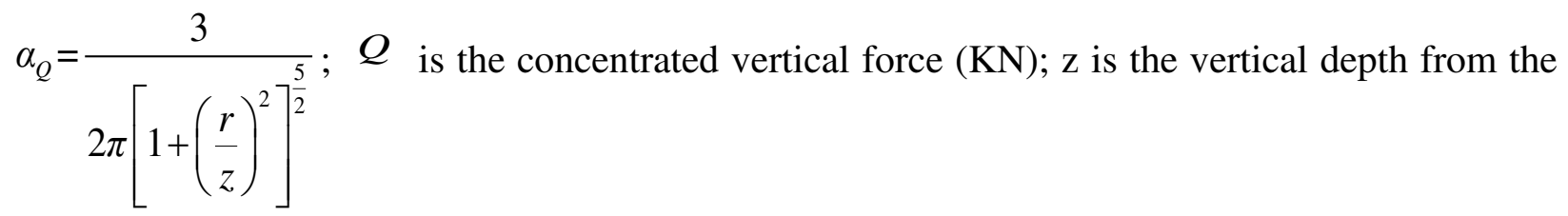

are the $n$ zeros of the Gauss-Legendre orthogonal polynomials, which can achieve an algebraic accuracy of $2 n+1$, and the weights of the nodes in the quadrature formula are all positive. Thus, the method has good numerical stability.

In summary, to overcome the limitations of the traditional methods of calculating the additional stress in foundation soils, the Gauss-Simpson Quadrature Algorithm for calculating the additional stress in foundation soils under an irregular load distribution that has an irregular distribution shape is proposed based on the Gauss-Simpson Quadrature Algorithm. The proposed method has a wide range of engineering applications. In this paper, we will elaborate upon the details of the Gauss-Simpson Quadrature Algorithm and its implementation.

\section{Additional Stress in Foundation Soil}

In the elastic theory, the additional stress in foundation soil is calculated based on the basic Boussinesq solution in elastic mechanics [3]:

$$
\sigma_{z}=\alpha_{Q} \frac{Q}{z^{2}}
$$

where $\alpha_{Q}$ is the coefficient of the additional vertical stress under a concentrated load; calculation point to the ground surface $(\mathrm{m})$. In spatial problems, $r$ is the horizontal distance from the computing points to the concentrated load point.

In engineering, there is no concentrated force, and the load is distributed. When the $r$ value of the calculation point is much larger than the boundary of the distributed load, the load can be replaced by a concentrated force in order to calculate the additional vertical stress. When the shape 
110 and size of the distributed load are not negligible, and there is a distributed load $P_{0}(x, y)$ on

111 foundation $\mathrm{A}$, the additional stress at any point $M(x, y)$ in the foundation can be obtained by

112 integrating over the load distribution area $A$ using Equation (1) [4]:

$113 \quad \sigma(x, y)=\iint_{A} \alpha_{Q} \frac{p_{0}(x, y)}{z^{2}} \mathrm{~d} x \mathrm{~d} y$

114 When $P_{0}(x, y)$ is a uniform load on a rectangular area, the double integral in Equation (2)

115 is integrable, and the settlement at each point can be expressed algebraically. However, when

$116 P_{0}(x, y)$ is irregular, the double integral in Equation (2) is generally non-integrable, i.e., the

117 foundation settlement under an irregular load distribution cannot be expressed directly in an 118 algebraic form.

119

2 Gauss-Simpson Quadrature Algorithm for Calculating the Additional Stress in Foundation

121 Soils

122

\subsection{Gaussian Quadrature Formula of the Double Integral [13-14]}

The double integral $\iint_{R} p(x, y) \mathrm{d} A$ is the volume enclosed by the surface $z=p(x, y)$ and

124 the plane R. For a rectangular region $R=\{(x, y) \mid a \leq x \leq b, c \leq y \leq d\}$, it can be written as a 125 superposition of two single integrals:

$126 \quad \iint_{R} p(x, y) \mathrm{d} x \mathrm{~d} y=\int_{a}^{b}\left(\int_{c}^{d} f(x, y) \mathrm{d} y\right) \mathrm{d} x$.

For a single integral in each layer $\int_{a}^{b} f(x) \mathrm{d} x$, the per-type interval transformation is

$128 x=\frac{b-a}{2} t+\frac{a+b}{2}$, such that the integration interval is $(-1,1)$. Then, 


$$
\int_{a}^{b} f(x) \mathrm{d} x=\frac{b-a}{2} \int_{-1}^{1} f\left(\frac{b-a}{2} t+\frac{a+b}{2}\right) \mathrm{d} t .
$$
can be used:

$$
\int_{-1}^{1} f(t) \mathrm{d} t \approx \sum_{k=0}^{n} A_{k} f\left(x_{k}\right) .
$$

133 Here, $A_{k}$ is the weight coefficients of the Gauss-Legendre quadrature formula (Table 1); $x_{k}$ is 134 the zero point of the orthogonal polynomial-Legendre polynomial, i.e., the Gaussian point in 135 Equation (5) (Table 1).

Table 1 Nodes and weight coefficients of the Gauss-Legendre quadrature formula ${ }^{[13-14]}$

From the Gaussian quadrature formula and Table 1, it can be seen that the quadrature weights and the Gauss node of the Gaussian quadrature formula are independent of the specific function, so they are only related to the function value of the integrand at the Gaussian point, which provides

142 insight into the Gauss-Simpson Quadrature Algorithm for calculating the additional stress in soils under a building foundation.

\subsection{Calculation of Additional Stress based on Gaussian Quadrature}

Because of the generality of the Gaussian quadrature formula, i.e., the independence of the quadrature formula from the specific functional form, the same number of quadrature nodes $(\mathrm{N})$

147 and weight coefficients can be used to calculate the double integral value of Eq. (2) for different 148 foundation structures while obtaining an algebraic accuracy of $2 n+1$. It is not limited by the type

149 of additional pressure at the bottom of the foundation $p_{0}(x, y)$ and the integrability of Equation 
150 (2). As the number of quadrature nodes increases, the additional stress keeps converging to the

151 exact value. Thus, the accuracy can be easily controlled.

152 The essence of the method is that the value of the additional pressure at the bottom of the

153 foundation at the point of Gaussian integration is interpolated, and then, the additional pressure at

154 the bottom of the foundation distribution curve is represented by the interpolated curve, and the

155 final approximation of the additional stress in a foundation under any additional pressure at the

156 bottom of the foundation is obtained through integration.

157 3. Calculation of Additional Stress in Three Types of Irregularly Shaped Foundations

$158 \quad 3.1$ Irregular Load Distribution with a Regular Distribution Shape

159

160

Fig. 1 The first case: an irregular load distribution with a regular distribution shape

161

162

Equation (2) is used for calculating the additional stress. Assuming that the length of a rectangular foundation is $l$ and the width is $b$, (e.g., as in Fig. 1) then Equation (2) can be written

164 as the superposition of two single integrals [13-14]: $\sigma(x, y)=\iint_{A} \alpha_{Q} \frac{p_{0}(x, y)}{z^{2}} \mathrm{~d} x \mathrm{~d} y$.

$$
\sigma(x, y)=\iint_{A} \alpha_{Q} \frac{p_{0}(x, y)}{z^{2}} \mathrm{~d} x \mathrm{~d} y
$$

165

$$
=\int_{-\frac{l}{2}}^{\frac{l}{2}}\left(\int_{-\frac{b}{2}}^{\frac{\mathrm{b}}{2}} \alpha_{Q} \frac{p_{0}(x, y)}{z^{2}} \mathrm{~d} x\right) \mathrm{d} y
$$

166 Assuming $x=\frac{b}{2} \cdot m$ and $y=\frac{l}{2} \cdot n$, then Equation (6) can be transformed as follows:

167

$$
\begin{aligned}
& \sigma(x, y)= \\
& \frac{b l}{4} \int_{-1}^{1}\left(\int_{-1}^{1} \alpha_{Q} \frac{p_{0}(0.5 b m, 0.5 \ln )}{z^{2}} \mathrm{~d} m\right) \mathrm{d} n
\end{aligned}
$$



$m_{i}$ and $n_{j}$, the additional stress is

$$
\sigma(x, y)=\frac{b l}{4} \sum_{i=0}^{k} \sum_{j=0}^{k} A_{i} A_{j}\left[\alpha_{Q} \frac{p_{0}(0.5 b m, 0.5 \ln )}{z^{2}}\right] .
$$

Where the additional pressure at the bottom of the foundation is

Equation (9) can be chosen in any functional type and substituted into algebraic Equation (8), which does not include the integral sign, and then, it can be solved analytically. integration points in each group are determined in the order of low to high accuracy and based on the number of integration points. For each group of integration point coordinates, the additional pressure at the bottom of the foundation at the integration point is obtained. Using the Gaussian quadrature formula, the integral values with different accuracies are derived, i.e., the additional stress values in the foundation with different accuracies. The minimum number of integration points to meet the accuracy requirement is obtained through comparison with the measurement data or the results of the traditional method. In practical engineering, we only need to ensure that the number of integration points is larger than the minimum number to obtain good results.

184 Moreover, the more the integration points, the higher the accuracy.

3.2 Irregular Load Distribution and Load Distribution Shape Characterized by Two Arbitrary Curves within Two Parallel Lines

Fig. 2 The second case: an irregular load distribution and a load distribution shape (Multiple perspectives of the art building itself and the foundation shape of the building) 
The method in this study is not limited to the calculation of additional stress in a foundation with a uniform load and regular shapes. (e.g., as in Fig. 2) For cases with irregular load 194 distributions and irregular shapes, an explicit algebraic equation for calculating the additional stress is given:

$$
S=\frac{b-a}{6}[f(a)+4 f(c)+f(b)]
$$

197 Here, $c=\frac{a+b}{2} ; a$ is the lower limit of integration; $b$ is the upper lower limit of integration; and $198 s$ is the Simpson integration formula value [13-14].

199 Equation (10) is the famous Simpson (Simpson) formula. A valuable feature of this equation 200 is that it introduces the relaxation factor $\frac{4}{6}=\frac{2}{3}$, which allows Simpson's formula to achieve a 201 higher algebraic accuracy than the trapezoidal formula with the same two integration points a 202 and $b$ [13-14]. In Simpson integration calculations, the relaxation technique is referred to as the 203 relaxation method, and it is a common method used in the modern analysis technique [13-14]. 204 Equation (10) appeared in the collection of papers that Simpson published in 1743, when the 205 relaxation technique was not yet systematically established and applied [13-14].

206 In multiple numerical integrations, the double integral over a non-rectangular region can be 207 approximated as rectangular regions by simply reducing it to a cumulative integral [13-14]:

$$
I=\int_{a}^{b} \int_{c(x)}^{d(x)} f(x, y) \mathrm{d} y \mathrm{~d} x
$$

Specifically, the explicit Simpson formula was used to transform the upper and lower limits of the inner curve of the double integral into an explicit functional expression for the integral limit 211 of the curve, thus transforming the implicit double integral in the formula for calculating the 
212 additional stress in a non-rectangular domain into an explicit cumulative integral by explicitly

213 removing the integral number, which is similar to the rectangular domain case used to obtain the

214 solution. The explicit analytical algebraic formula for the approximation of additional stress is

215 obtained in a manner similar to the rectangular domain case.

$$
\begin{aligned}
& I \approx \int_{a}^{b} \frac{k(x)}{3}[f(x, c(x))+ \\
& 4 f(x, c(x)+k(x))+f(x, d(x))] \mathrm{d} x
\end{aligned}
$$

217 Here, $k(x)=\frac{d(x)-c(x)}{2}$. Then, the Gaussian quadrature formula is used for each integral, and 218 the approximation of the integral $I$ is obtained.

\subsection{Irregular Load Distribution and Irregular Distribution Shape}

Fig. 3 The third case: an irregular load distribution and an irregular distribution shape that

\section{satisfies certain conditions}

In the case shown in Fig. 3, the loads are irregularly distributed, but they can be expressed analytically, and the load is distributed in an irregular shape that satisfies certain conditions.

$$
I=\int_{a(x)}^{b(x)} \int_{c(x)}^{d(x)} f(x, y) \cdot \mathrm{d} x \mathrm{~d} y
$$

For Equation (13), where both the inner and outer layers are double integrals of the upper and

229 lower limits of the curve and the additional pressure at the bottom of the foundation is an irregular

230 load distribution, the problem is transformed into calculating the volume of a three-dimensional

231 flat-bottomed surface enclosed by a curved integral domain $\left\{\begin{array}{l}a(x)-b(x) \\ c(x)-d(x)\end{array}\right.$ with a height of 

$z=f(x, y)$. Using Simpson's formula and Gaussian quadrature, the double integral of an irregularly loaded, irregularly shaped region is transformed into an explicit algebraic expression.

234 Assuming that the boundary of the curved integral domain $\left\{\begin{array}{l}a(x)-b(x) \\ c(x)-d(x)\end{array}\right.$ in the y-direction 235 is $\left[c_{\min }, d_{\max }\right]$, where $c_{\min }$, and $d_{\max }$ are constants, nodes $\mathrm{c}$ and $\mathrm{d}$ exist in the y-direction of the 236 integral domain, between $[c, d]$ and $\left[c_{\min }, c\right],\left[d, d_{\max }\right]$. The integral limits $a(x)-b(x)$ in the $237 x$ direction in Equation (13) can be represented analytically by the same function. Such integral 238 domains are common in the widely adopted in circular and square art architectures. Under this 239 premise, Equation (13) can be converted to

$$
\begin{aligned}
& I=\int_{a(x)}^{b(x)} \int_{c(x)}^{d(x)} f(x, y) \cdot \mathrm{d} x \mathrm{~d} y \\
& =\int_{a(x)}^{b(x)} \int_{c_{\min }}^{c} f_{1-2}(x, y) \cdot \mathrm{d} x \mathrm{~d} y+\int_{a(x)}^{b(x)} \int_{c}^{d} f_{2-3}(x, y) \cdot \mathrm{d} x \mathrm{~d} y+\int_{a(x)}^{b(x)} \int_{d}^{d_{\max }} f_{3-4}(x, y) \cdot \mathrm{d} x \mathrm{~d} y \\
& =\int_{c_{\min }}^{c} \int_{a(x)}^{b(x)} f_{1}(x, y) \cdot \mathrm{d} x \mathrm{~d} y+\int_{c}^{d} \int_{a(x)}^{b(x)} f_{2}(x, y) \cdot \mathrm{d} x \mathrm{~d} y+\int_{d}^{d_{\max }} \int_{a(x)}^{b(x)} f_{3}(x, y) \cdot \mathrm{d} x \mathrm{~d} y \\
& =I_{1}+I_{2}+I_{3}
\end{aligned}
$$

\section{For}

$$
\begin{aligned}
& I_{1}=\int_{c_{\min }}^{c} \int_{a(x)}^{b(x)} f_{1}(x, y) \cdot \mathrm{d} x \mathrm{~d} y \\
& I_{2}=\int_{c}^{d} \int_{a(x)}^{b(x)} f_{2}(x, y) \cdot \mathrm{d} x \mathrm{~d} y, \\
& I_{3}=\int_{d}^{d_{\max }} \int_{a(x)}^{b(x)} f_{3}(x, y) \cdot \mathrm{d} x \mathrm{~d} y
\end{aligned}
$$

243 Simpson's formula is used to achieve explicitation of the inner integral variable limit integrals, 244 and then the Gauss-Legendre formula is used to deal with each integral's constant limit integral.

\subsection{Summary}

Based on the above analysis, the method proposed in this study is not limited to the calculation 
long as the corresponding double integral in the equation is converted into a cumulative integral

249 following the above method, the explicit expression for the additional stress can be obtained in a 250 manner similar to that for the rectangular domain case.

251 In engineering applications, only the location of the interpolation points of the Gauss252 Legendre formula, the interpolated weight coefficients, and the specific type of Simpson's formula 253 are needed for the field engineers to obtain the additional stress manually, without complex 254 integration and interpolation. This is nearly impossible for traditional methods and finite element 255 methods. Therefore, the proposed method has excellent potential for broad application.

\section{4. Validation of Traditional Elasticity Theory-Based Methods}

$p_{0}$ has an analytical solution in traditional elastic theory [4]:

$$
\begin{aligned}
& \sigma_{z}=\frac{3 p_{0}}{2 \pi} \int_{0}^{l} \int_{0}^{b} \frac{z^{3}}{\left(x^{2}+y^{2}+z^{2}\right)^{\frac{5}{2}}} \mathrm{~d} x \mathrm{~d} y \\
& =\frac{p_{0}}{2 \pi}\left[\arctan \frac{n^{\prime}}{m \sqrt{1+m^{2}+n^{\prime 2}}}+\right. \\
& \left.\frac{m n^{\prime}}{\sqrt{1+m^{2}+n^{\prime 2}}}\left(\frac{1}{m^{2}+n^{\prime 2}}+\frac{1}{1+m^{2}}\right)\right] \\
& =\alpha_{c} p_{0}
\end{aligned}
$$

Here, $m$ is the ratio of the depth of the calculated point $z$ to the width of the load $b ; n^{\prime}=l / b$;

$261 n^{\prime}$ is the ratio of the length of the rectangular foundation $I$ to the width $b$; and $\alpha_{c}$ is the 262 coefficient of the additional vertical stress at the corner points of the uniformly distributed 263 rectangular load, which can be looked up in the table based on $l / b$ and $\mathrm{z} / \mathrm{b}$.

264 In Equation (16), the numerical expression for the coefficient of the additional stress is 


$$
\alpha_{c}=\frac{b l}{4} \cdot \sum_{i=0}^{k} \sum_{j=0}^{k} A_{i} A_{j} \frac{\alpha_{Q}}{z^{2}} .
$$

The nodes and coefficients of the Gauss-Legendre quadrature formula when $n=2,3,4$, and 5 are used to form Equation (16). The values of the corner point influence coefficients are calculated when $l / b$ takes various values. Then, the result is compared with the analytical solution to verify the validity of the Gauss-Simpson Quadrature Algorithm and to determine the minimum number of nodes.

Figure 4 shows the comparison between the Gaussian quadrature formulas of different orders and the traditional elastic theory method in terms of the coefficient of the additional stress at the corner points of a rectangular foundation. It can be seen that as the number of quadrature nodes increases, the accuracy of the Gaussian quadrature formula increases accordingly and approaches the analytical solution for the elastic theory. As can be seen from Figure 4, the approximation value calculated using Gaussian quadrature is smaller than the analytical solution of the elasticity theory, which converges continuously from the lower limit of the analytical solution. Therefore, using Gaussian quadrature to calculate the additional stress is similar to using the elastic theory method. This is related to the concavity of the integrand in Equation (2) and the interpolation characteristics at the integration points.

\section{Fig. 4 Comparison of the additional stress calculated using the Gaussian quadrature method}

\section{and influence coefficients of the additional stress of different orders}

It should be noted that the Gaussian quadrature formula has a high algebraic accuracy of $2 n+1$

for the same number of quadrature nodes, and the quadrature nodes are 0 points of orthogonal polynomials. The node weights in the quadrature formula are all positive and have a high degree of numerical stability. When the order of the quadrature formula exceeds five, it is not economical 
to improve the computational accuracy simply by increasing the number of quadrature nodes. The advantage of Equation (8) in calculating the additional stress is that the calculation formula can be expressed analytically and the calculation process can be solved algebraically, generally by hand.

291 One disadvantage of the method is that the accuracy of the calculation can only be as good as the 292 5th order Gauss-Legendre quadrature formula. To further improve the calculation accuracy of 293 Equation (2), the complex quadrature formula can be used. That is, the interval can be divided into several equal parts, and each part can be solved separately using the Gaussian quadrature formula, which effectively improves the calculation accuracy.

Figure 5 illustrates the calculation of the coefficient of the additional stress using the complex quadrature formula and the traditional elastic theory method, the complex quadrature formula does improve the calculation accuracy. The curve basically overlaps with that of the traditional elastic theory method. The complex Gaussian quadrature formula is computationally intensive and can be implemented using computer programming. In this study, Matlab was used to program the solving process for the dual complex Gauss-Legendre quadrature formula [13].

Fig. 5 Comparison of the additional stress calculated using the complex Gaussian quadrature method and 5 orders of the Gaussian quadrature method

\section{Comparison with the FE simulation Results}

Taking the first case in Section 3 as an example, the simulation results of the proposed method and the finite element simulation are compared. The short side $b$ is the $x$ axis, and the long side

$309 l$ is the $y$ axis. The uniform load is a three-dimensional surface $z=\cos \left(\frac{\pi}{400} x\right)$ on the base $(b \times l)$, 310 and $(-200 \leq x \leq 200,-l \leq y \leq l)$ ). For $b=400$, the maximum value of the load 
$311 z=\cos \left(\frac{\pi}{400} \cdot 0\right)=1 \mathrm{MPa}$ occurs at $x=0$. The Poisson's ratio of the soil beneath the foundation is

$312 v=0.25$, and $E_{0}=5 \mathrm{MPa}$. When $\frac{l}{b}$ has different values, a finite element model is established

313 to investigate the difference between the simulation results of the additional stress and the results

314 of the method proposed in this study.

315 The Adina software was used for the finite element simulation. Three dimensional solid 316 elements were used for the model, and the bottom surface of the model was fixed. The load was

317 applied using the spatial function, which defined a point array of two-dimensional spatial 318 distribution surfaces and was used to define the load used to generate the cosine surface load with 319 a rectangular base that is parallel to the $y$ axis. Figure 6 shows the finite element model and the 320 load function.

Fig. 6 The finite element method model

As can be seen from Figure 7, the FE method results are generally consistent with those of the 325 Gauss-Simpson Quadrature Algorithm in terms of the magnitude, but there are localized 326 differences in the curve patterns. The curve direction and the shape of the Gaussian quadrature 327 formula method are very similar to the curve for a uniform load. The FE simulation curve is 328 initially smaller than or similar to that of the Gaussian quadrature, and then it becomes larger than 329 the Gaussian quadrature. This is due to the boundary conditions of the FE method model. During 330 the simulation, the boundary conditions were applied on the bottom surface of the 3D model, and 331 no constraints were applied on the sides. Therefore, when the long side $l$ is small, the loading 332 element is at the center of the top surface of the 3D model, which is constrained by the surrounding 
333 elements, i.e., the completely confined condition. Therefore, the FE method results are similar to

334 or smaller than the results of the Gaussian quadrature. When the long side $l$ is large, the model size

335 and the number of elements are limited by the computer's memory, the rectangular base corner

336 points are closer to the model boundary, and since there are no constraints on the sides of the 3D

337 model, i.e., the unconfined condition, the additional stress becomes very large.

Fig. 7 Comparison of the additional stress calculated using the Gaussian quadrature method and the FE simulation method under an irregular cosine load

This comparison shows that the Gaussian quadrature formula is more concise and practical,

343 its results are more reasonable, and the calculation is more controllable. In contrast, the FE 344 simulation is computationally intensive, complex to implement and operate; the calculation results 345 are limited by the model, load, and boundary conditions; and it is hard to control the reasonableness 346 of the calculation results.

\section{Additional Stress Calculation for A Tower Foundation in the Dongrong Mining Area}

The Dongrong mine is located in the northern succession area of the Shuangyashan Mining

350 situated on the first-grade terrace of the Songhua River and belongs to the river floodplain phase.

351 The soil is composed of a clay layer (0.5-16 m thick) and a sand layer (about $40 \mathrm{~m}$ ) composed of 352 Holocene (Q4) alluvium, which is a typical binary structure. The groundwater is phreatic water, 353 and the water in the lower sand layer is pressurized water and is abundant. The upper phreatic 354 water is just $0.5-1 \mathrm{~m}$ below the surface, and the water is not harmful to any kind of concrete. The 355 standard freezing depth is $2.20 \mathrm{~m}$. The Quaternary and Tertiary strata directly overlie the coal strata, 
so a special freezing method has to be used in the new mine to construct and drill the shaft. The first completed mine in the Dongrong mining area, Dongrong mine No.2, has a design capacity of 1.5 million t/a. The main shaft tower of the mine was built on special artificial freeze-thaw soil (general soil and sandy soil from the quaternary alluvium) in the frozen shaft.

The proposed method was used to calculate the additional stress in the foundation of the main shaft tower of Dongrong mine No.2. The tower's foundation is rectangular, with a length of $l=4 \mathrm{~m}$ and a width of $b=3.2 \mathrm{~m}$. The foundation is buried $1 \mathrm{~m}$ deep, and the designed bottom is $0.6 \mathrm{~m}$ from the natural bottom. The modulus of the deformation of the foundation soil is $E_{0}=5.6 \mathrm{MPa}$, the Poisson's ratio is $v=0.4$, and the unit weight is $\gamma=19.8 \mathrm{kN} / \mathrm{m}^{3}$. The load is equivalent to $z=0.09375 \cdot \cos \left(\frac{\pi}{3.2} x\right)(\mathrm{MPa})(-1.6 \leq x \leq 1.6,-2 \leq y \leq 2)$.

The following is the code for the dual complex Gauss-Legendre quadrature formula. Here, $\mathrm{n}$ $=2$ (i.e., 3 nodes) and there are 10 intervals [13].

The $\mathrm{M}$ file is as follows, in which the lines starting with\% are descriptive text only, and do not participate in the program's operation.

$\% f$ is the pre-defined integrand; and $\% \quad a_{1}$ and $a_{2}$ are the upper and lower integration limits of the outer layer of the double integral.

$\% b_{1}$ and $b_{2}$ are the upper and lower integration limits of the inner layer of the double integral; and $\% m$ is the order of integration.

$\% \quad n_{1}$ is the number of inner complex intervals of the double integral; and $\% n_{2}$ is the number of outer complex intervals of the double integral.

$\% r$ is the aspect ratio of the rectangular foundation; $\% h_{1}$ is the length of the inner complex 
377 interval; and $\% h_{2}$ is the length of the outer complex interval.

$378 \% t$ is the Gauss-Legendre integration points; and $\% A$ is the Gauss-Legendre integration 379 weight.

380 The following is the specific function field: 


$$
\begin{aligned}
& \text { \%Function start } \\
& \text { function } \\
& \begin{aligned}
s= & \text { ch }_{2} \text { Gauss_Legendre } \\
2 & \left(f, a_{1}, b_{1}, a_{2}, b_{2}, m, n_{1}, n_{2}, r\right) \\
h_{1} & =\frac{\left(b_{1}-a_{1}\right)}{n_{1}} \\
h_{2} & =\frac{\left(b_{2}-a_{2}\right)}{n_{2}} \\
s & =0 \\
t & =\left[-\sqrt{\left(\frac{3}{5}\right)}, 0, \sqrt{\left(\frac{3}{5}\right)}\right] \\
A & =\left[\frac{5}{9}, \frac{8}{9}, \frac{5}{9}\right] ; \\
& \% \text { Layer } 1-1 \text { cycle starts }
\end{aligned}
\end{aligned}
$$$$
\text { for } l=1: n_{2}
$$

\%Layer 1-2 cycle starts

for $l=1: n_{1}$

\%Layer 2 cycle starts

$$
\begin{gathered}
x=\frac{h_{1} \times t}{2}+a_{1}+\left(k-\frac{1}{2}\right) \times h_{1} ; \\
y=\frac{h_{2} \times t}{2}+a_{2}+\left(l-\frac{1}{2}\right) * h_{2} ; \\
\text { \%Layer } 3-1 \text { cycle starts } \\
i=1: m+1
\end{gathered}
$$

\%Layer 3-2 cycle starts

$$
j=1: m+1
$$

$$
\begin{aligned}
& F=\operatorname{feval}(f, x(i), y(j), r) ; \\
& s=s+A(i) \cdot A(j) \cdot F ;
\end{aligned}
$$

$\%$ End of layer 3-2 loop

end

$\%$ End of layer 3-1 loop

end

\section{$\%$ End of layer 2 loop}

$$
\begin{aligned}
& \text { end } \\
& \text { \%End of layer 1-2 loop } \\
& \text { end } \\
& s=s * h 1 * h 2 / 4 ; \\
& \% \text { End of layer 1-1 loop } \\
& \text { end }
\end{aligned}
$$

$\%$ End of function 


$$
\begin{aligned}
& f=
\end{aligned}
$$

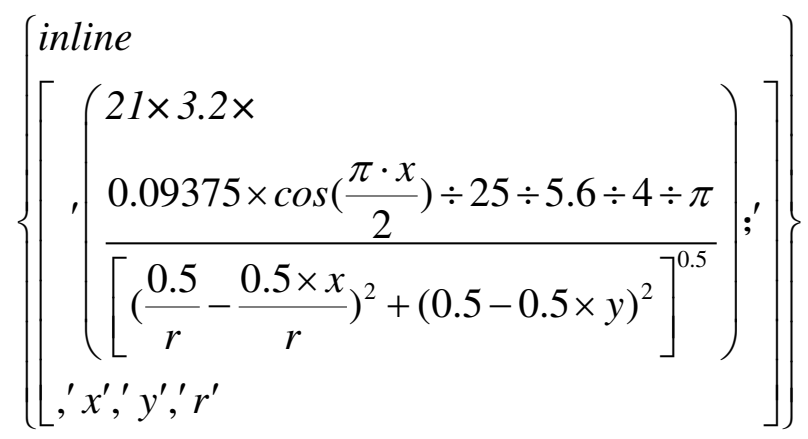

$$
\begin{aligned}
& m=2 \text {; } \\
& n_{1}=10 ; n_{2}=10 \text {; }
\end{aligned}
$$

The solution command is $r=1.25$;

$$
\begin{aligned}
& a_{1}=-1 ; a_{2}=-1 ; \\
& b_{1}=1 ; b_{2}=1 ; \\
& {\left[\begin{array}{l}
q u a d=c h_{2} \text { Gauss_Legendre } \\
\left(f, a_{1}, b_{1}, a_{2}, b_{2}, m, n_{1}, n_{2}, r\right) ;
\end{array}\right]} \\
& \text { quad =vpa }(q u a d, 10)
\end{aligned}
$$

The result is $0.01626499813(\mathrm{MPa})$, which is smaller than the value we measured at the construction site.

\section{Conclusions}

In order to improve the calculation method for the additional stress in an irregularly shaped

387 foundation under an irregular load distribution, a calculation method, i.e., the Gauss-Simpson Quadrature Algorithm, which is based on elasticity theory, the Gauss-Legendre quadrature formula, and the Simpson quadrature formula, was proposed. The results were compared with that of the traditional elastic theory and an FE simulation, as well as with the field measurement data for the foundation of a shaft tower in Dongrong mine No.2.

The conclusions are as follows.

(1) For the same integration points $(n)$, the finite element method can only reach an algebraic 394 accuracy of $n$, whereas the Gauss-Legendre quadrature uses the zeros of Legendre's orthogonal polynomials as interpolation points and can reach an algebraic accuracy of $2 n+1$. This is of great 
importance for decreasing the computational cost, improving the computational efficiency, and increasing the computational accuracy.

(2) For irregular load distributions, the number of interpolations in the finite element method cannot be too high, and the element order cannot be too high due to the Runge phenomenon, which can lead to mesh distortion and numerical instability. Using a large number of low-order elements can greatly increase computational time. The Gauss-Legendre quadrature formula has positive integration weights and high numerical stability, which is one of its advantages over the finite element method.

(3) The biggest advantage of the Gaussian quadrature is that the explicit analytical algebraic equation for the additional stress in an irregularly shaped foundation under any irregular load distribution can be obtained, while avoiding numerical operations such as interpolation, integration, and solving large-scale linear systems of equations. The FE method usually require a generalpurpose calculation software for execution, which is expensive and difficult to develop. Therefore, it is not suitable for application in practical engineering.

(4) For an irregular load distribution in a rectangular domain, the additional stress can be directly obtained using Table 1, that is, the explicit analytical algebraic expression of the integral value. The higher the order, the more terms in the algebraic equation. In general, a satisfactory accuracy can be achieved when $n<5$. Moreover, the nodal positions and weight coefficients in shaped distributions and irregular load distributions. Simpson's quadrature formula utilizes the same number of quadrature nodes as the trapezoidal formula, but it yields higher algebraic accuracy than the trapezoidal formula. Simpson's formula is used to transform the upper and lower 
419 integrals of the inner curve of the double integral into a $1^{\text {st }}$ order explicit expression for the integral

420 limit of the curve. Then, Simpson's formula is applied once again to the outer integral, thus

421 transforming both the inner and outer integrals of the integral limit of the curve into the sum of 422 singular integrals with several constant integral limits. Thereafter, the Gauss-Legendre quadrature 423 formula is applied to obtain the explicit analytical algebraic formula of the additional stress in an 424 irregularly shaped foundation under any irregular load distribution.

(6) The calculation of the additional stress under three conditions was discussed. The first is an irregular load distribution and a regular distribution shape (e.g., rectangular or circular). The second is an irregular load distribution and a load distribution shape characterized by two arbitrary curves within two parallel lines. The third is an irregular load distribution and an irregular 429 distribution shape. Basically, these three conditions cover almost all possible engineering 430 applications. method can meet the requirements in most cases, for the sake of theoretical perfection and rigor, we studied the calculation accuracy of the Gaussian quadrature formula beyond the 5 th order. Since

434 the improvement of the calculation accuracy was not obvious in this case, we introduced the

436 which may not be suitable for direct field applications. reasonable request. 
This work was supported by the Youth Nursery Project of the Liaoning Provincial Education

442 Department: [grant numbers: LJ2020QNL010]. and the Discipline Innovation Team of Liaoning Technical 443 University [grant number: LNTU20TD08].

\section{Acknowledgments}

We thank LetPub (www.letpub.com) for its linguistic assistance during the preparation of this 446 manuscript.

\section{REFERENCES}

[1] QIU Yu, QIAN Guo-chao, LIU Song-yu. DJM pile treated soft clay foundation on Express highway the analysis of the additional stress in composite foundation and the settlement of composite foundation[J]. Journal of Highway and Transportantion Research and Development, 2001, 18(01): 1-5.

[2] CAO Wen-gui, DENG Xiang-jun, ZHANG Chao. Layerwise summation method for ground foundation

454 [3] XU Zhi-lun. Elasticity [M]. ed.4th. Beijing: Higher Education Press, 2006: 296-297.

455 [4] ZHANG Xiang-dong,LI Zhang-zhen,LI Ping.Soil mechanics[M].ed.1.Beijing:China Communications Press,2006: 104-105.

457 [5] Winterkorn, Hans Friedrich and Fang, Hsai-yang: Foundation Engineering Handbook, New York, 1975.

458 [6] H.Y.Huang: Influence Charts for Two-Layar Elastic Foundation, A.S.C.E. Vol.95, NoSM2, March 1969, 
[7] LUO Shi-yi. The Calculation of Additional Stress and Settlement for the Foundation of Multilayered Horizontal Soil [D]. Xian: Chang'an University2003

[8] ZHANG Jia-fan, ZHANG Hui-mei. Additional stress in pavement structure due to asymmetrical settlement of soft subgrade[J]. Journal of Chang'an University(Natural Science Edition), 2003, 23(03): $21-25$.

[9] GAO Xin-wen. Analysis of additional stresses in asphalt pavement due to asymmetrical subsidence of Mined-out region[J]. Journal of Highway and Transportation Research and Development, 2016, 33(10):

[10] GUO Run-hua, LING Jian-ming. Additional stresses of pavement subgrade resulted from aircraft

[11] WANG Xin-quan, ZHANG Jiang, ZHANG Shi-min, et al. A study of the additional stress caused by the tip resistance of a Y-shape pile with considering abnormality effect[J]. Rock and Soil Mechanics, 2016, 37(05): 1268-1280.

[12] LV Ya-ru, LIU Han-long, WANG Xin-quan, et al. A MODIFIED GEDDES'S SOLUTION FOR FOUNDATION ADDITIONAL STRESS INDUCED BY X-SECTION PILE[J]. Chinese Journal of Rock Mechanics and Engineering, 2013, 32(02): 349-362.

[13] ZENG Fan-hui.Numerical analysis[M]. ed.1. Xuzhou: China University of Mining and Technology press, 2009: 200-201.

[14] LI Qing-yang, WANG Neng-chao, YI Da-yi.Numerical analysis[M]. ed. 5th. Beijing: Tsinghua University Press, 2008: 126-128. 


\begin{tabular}{|c|c|c|}
\hline$n$ & $x_{k}$ & $A_{k}$ \\
\hline 0 & 0.0000000 & 2.0000000 \\
\hline 1 & \pm 0.5773503 & 1.0000000 \\
\hline \multirow{2}{*}{2} & \pm 0.7745967 & 0.5555556 \\
\hline & 0.0000000 & 0.8888889 \\
\hline \multirow{2}{*}{3} & \pm 0.8611363 & 0.3478548 \\
\hline & \pm 0.3399810 & 0.6521452 \\
\hline \multirow{3}{*}{4} & \pm 0.9061798 & 0.2369269 \\
\hline & \pm 0.5384693 & 0.4786287 \\
\hline & 0.0000000 & 0.5688889 \\
\hline \multirow{3}{*}{5} & \pm 0.9324695 & 0.1713245 \\
\hline & \pm 0.6612094 & 0.3607616 \\
\hline & \pm 0.2386192 & 0.4679139 \\
\hline
\end{tabular}




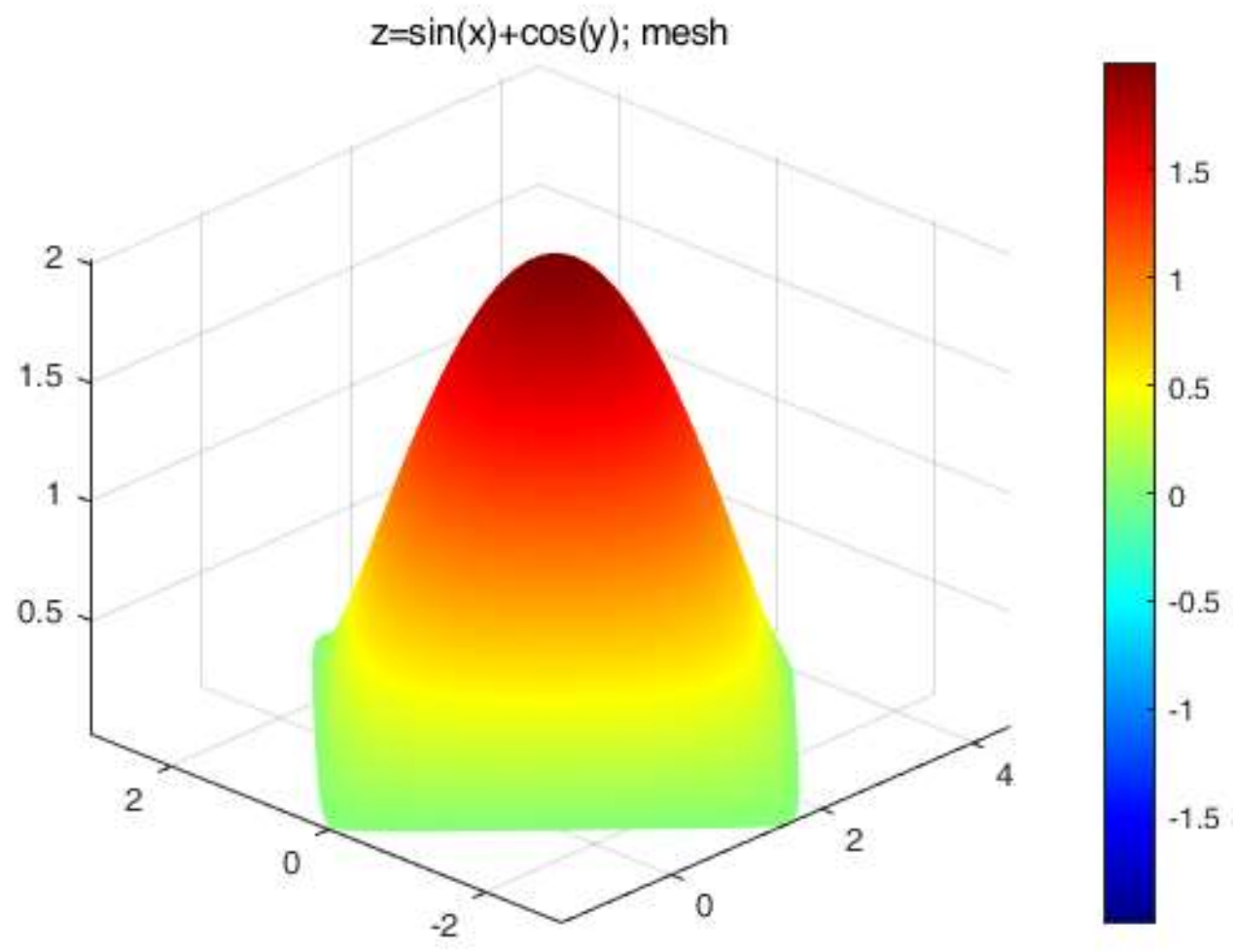

483 

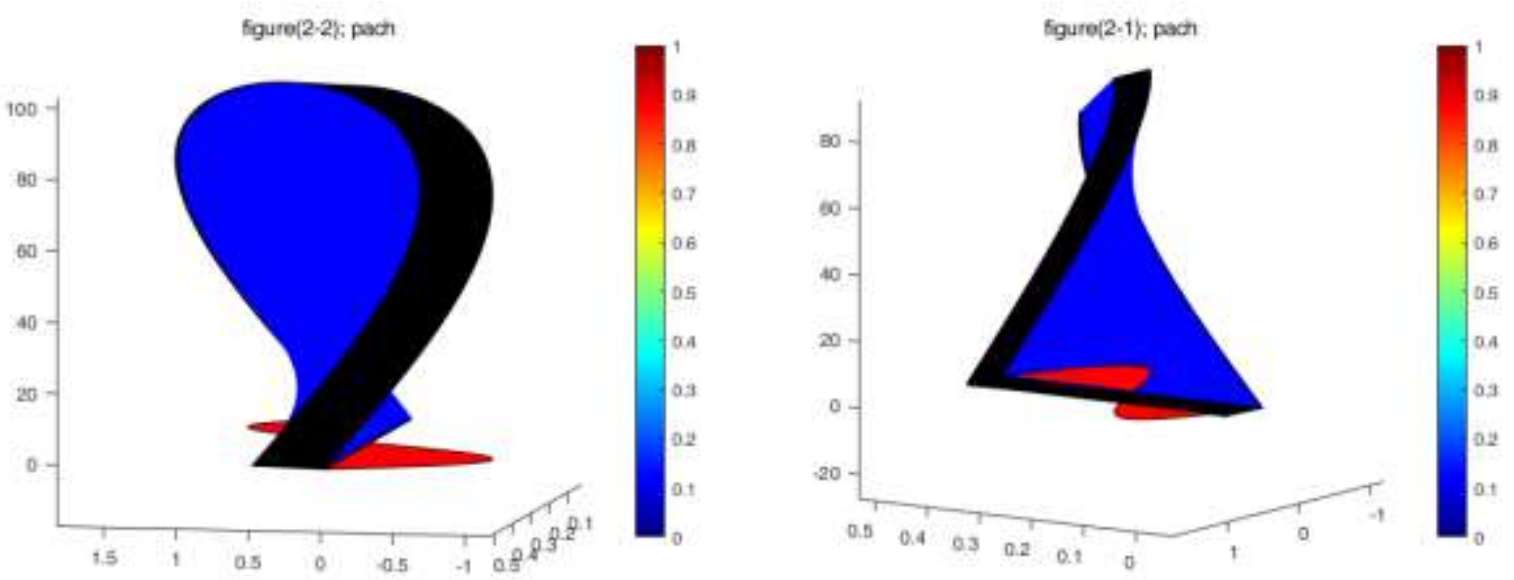

486
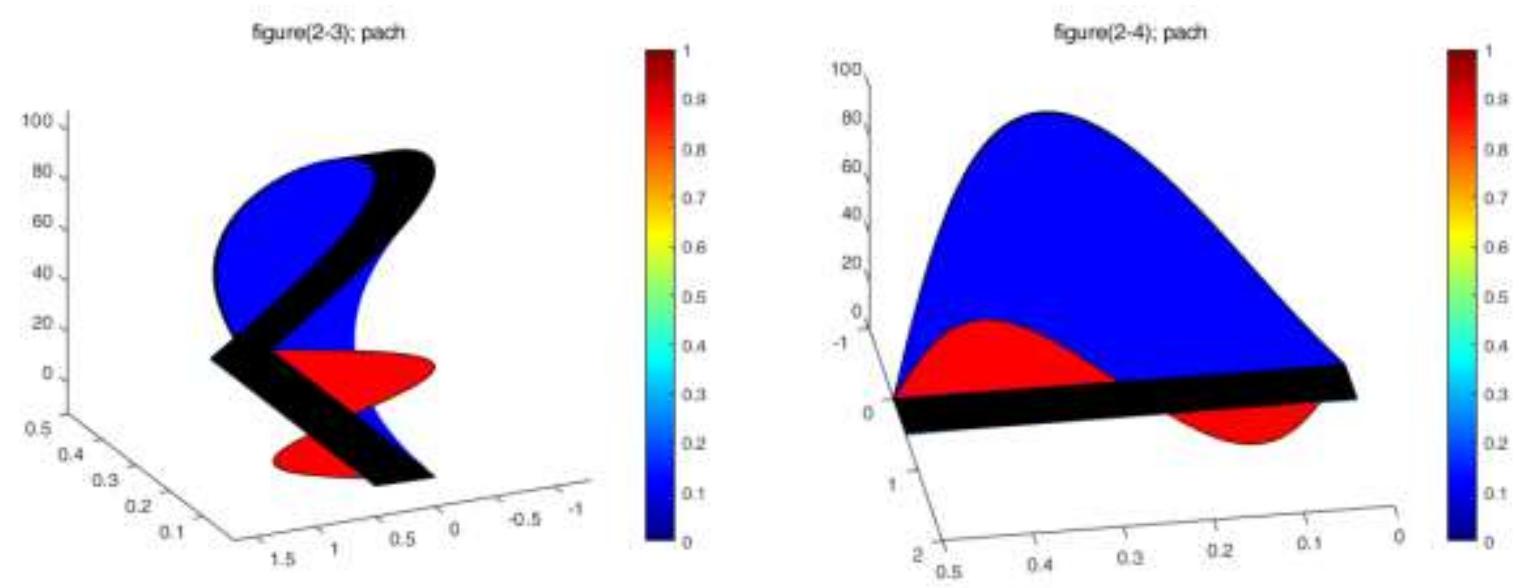

Fig. 2 The second case: an irregular load distribution and a load distribution shape characterized by two arbitrary curves within two parallel lines

490 (Multiple perspectives of the art building itself and the foundation shape of the building) 


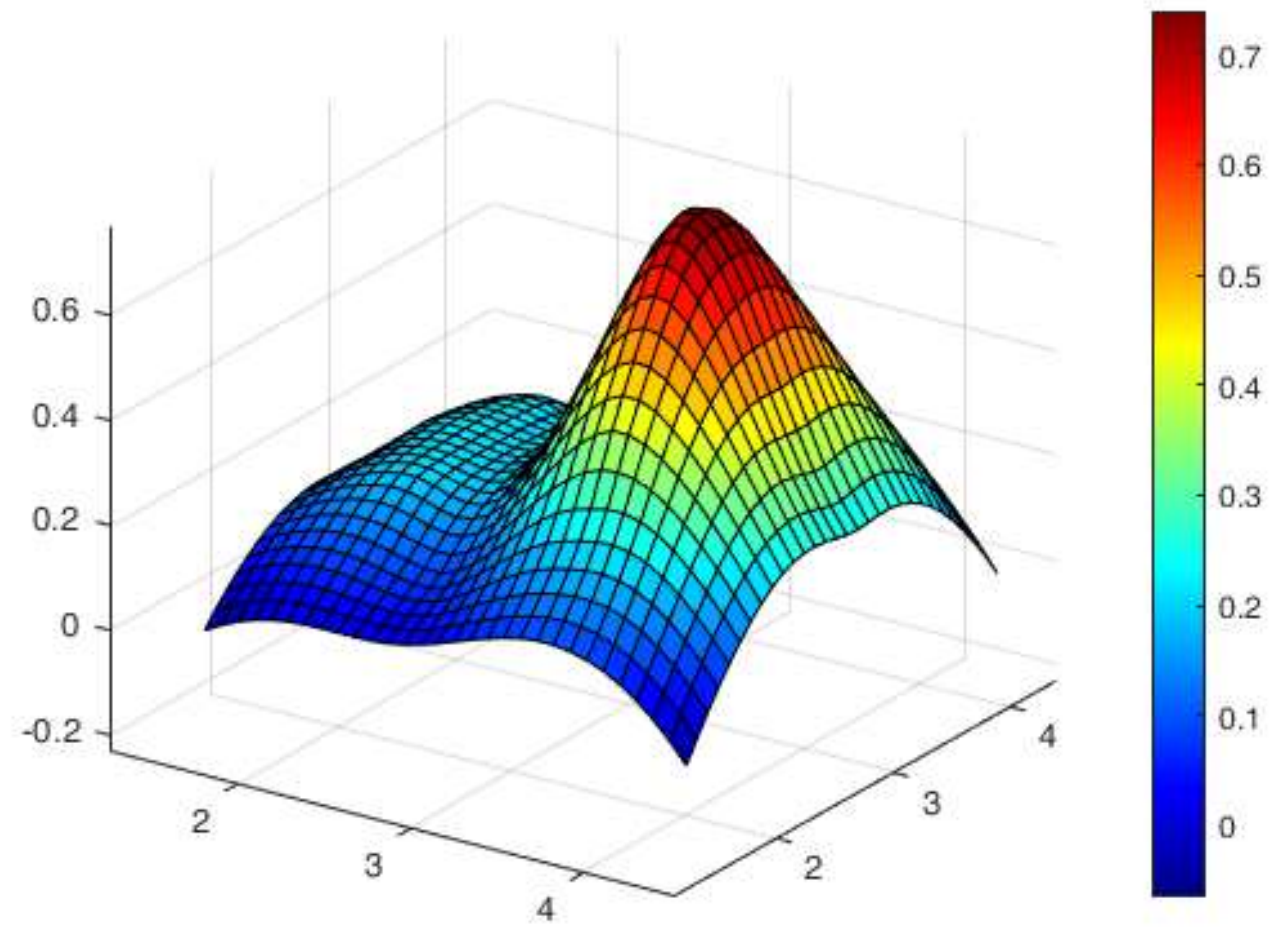




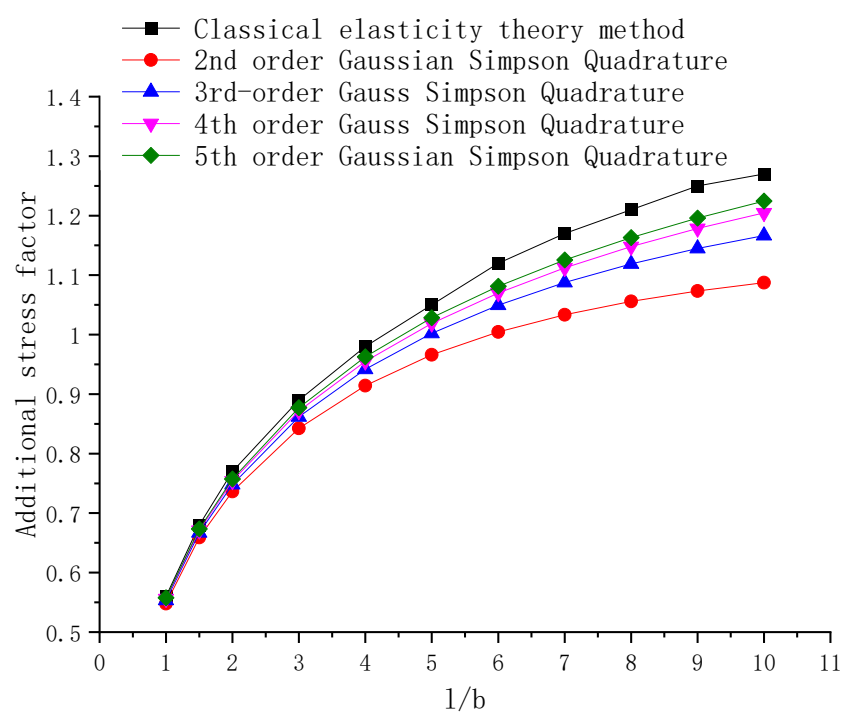

496 


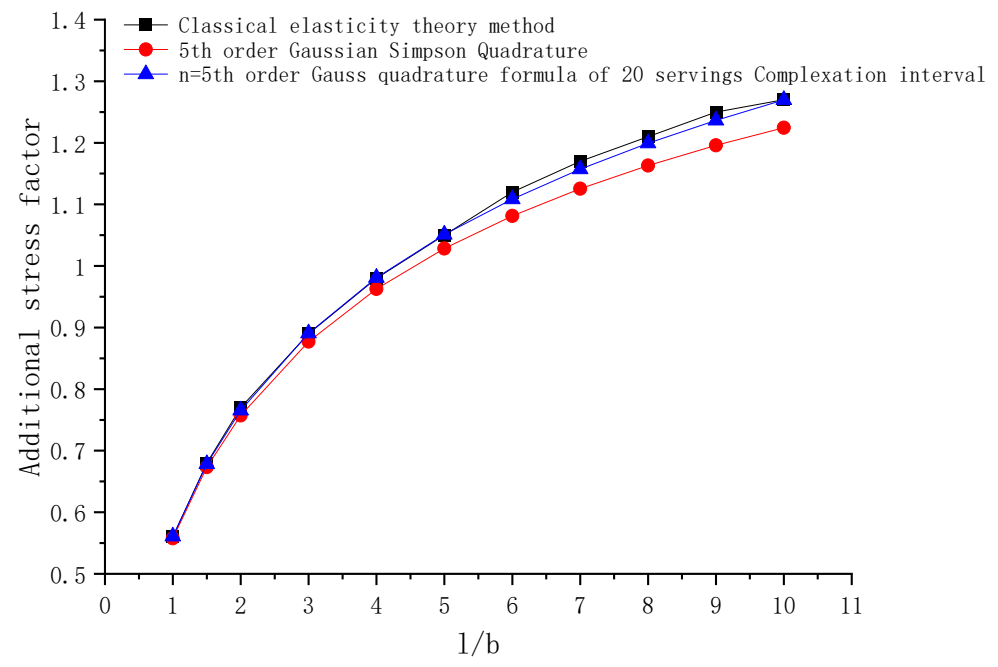




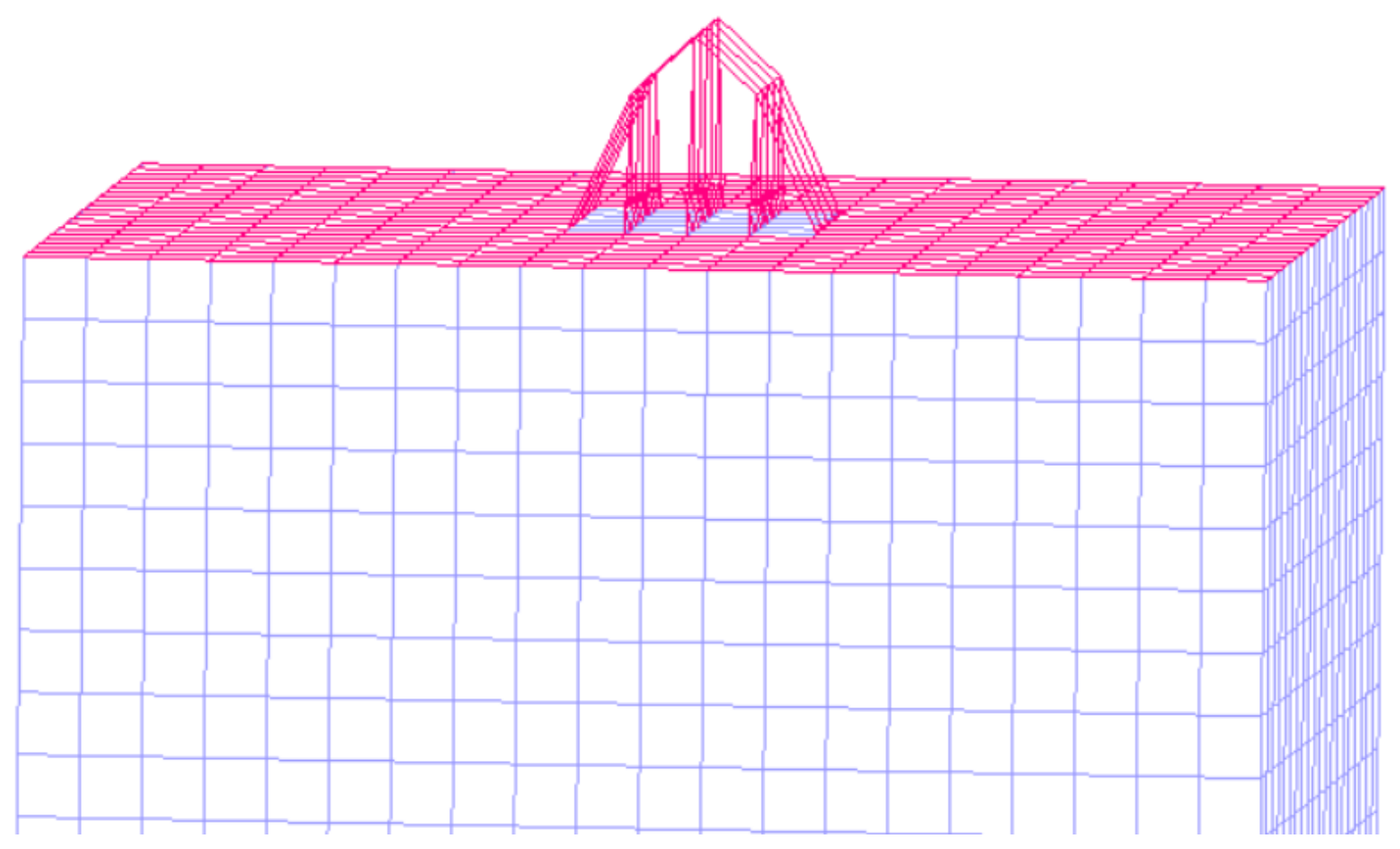




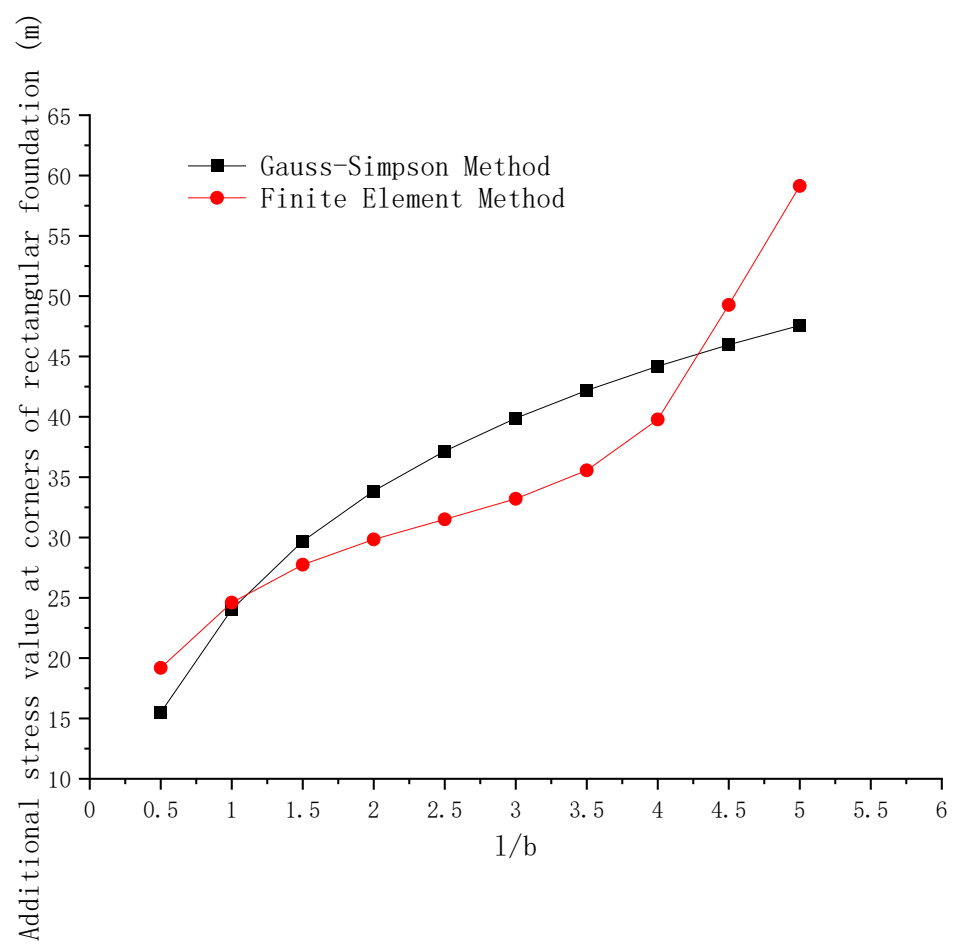

